\title{
Deuteron spin-flip resonance widths and the spin response function
}

\author{
S. R. Mane \\ Convergent Computing Inc., P.O. Box 561, Shoreham, New York 11786, USA
}

(Received 12 September 2007; published 7 November 2007)

\begin{abstract}
The spin response function is used to analyze the spin-flip resonance widths of stored polarized deuteron beams. It is found, using simple model assumptions, that the contribution of the vertical betatron oscillations (for an rf radial dipole field spin-flipper) reduces the resonance width by an amount in good agreement with recent measurements. It is also noted that, for spin-flip measurements with an rf-solenoid spin flipper, the spin response formalism also yields an answer consistent with experimental data.
\end{abstract}

DOI: 10.1103/PhysRevSTAB.10.111001

PACS numbers: 29.27.Hj, 29.20.Dh, 02.60.Lj, 02.30.Nw

The spin flip of stored particle beams in situ is a valuable experimental tool. The spin flip is typically accomplished by sweeping the frequency of an rf dipole or rf solenoid (a "spin flipper") across the frequency of a depolarizing resonance. (In practice, the spin flipper frequency is aliased with a harmonic of the beam circulation frequency.) Theoretically, the process can be analyzed using the Froissart-Stora formula [1]. The formula was originally derived for use in synchrotrons where the beam energy is swept across the (fixed) frequency of a depolarizing resonance. However, some care must be exercised when using the Froissart-Stora formula to analyze the action of a spin flipper; if the device is an rf dipole, then the "spin" flipper can also excite vertical betatron oscillations, these oscillations in turn can couple to the spins and decohere them (i.e. depolarize the beam). This was noted, for example, at LEP [2], where the (inadvertent) excitation of vertical betatron oscillations during resonant depolarization measurements was observed and caused the direction of the backscattered photons to jump around, distorting the polarimeter readings. In this paper, I shall analyze a simple model of the spin flip of a stored polarized beam, including the coupling of the rf-dipole field to the vertical betatron oscillations, to derive a simple expression for the "effective" resonance strength. I shall employ the "spin response function" formalism developed by Kondratenko [3]. Kondratenko actually solved for the perturbation to the $\boldsymbol{n}$-axis, where he assumed that $\boldsymbol{n}_{0}$ (the unperturbed closed-orbit solution) was vertical. However, the core of Kondratenko's formalism is applicable to the analysis of the spin-orbit coupling induced by the coherent vertical betatron oscillations driven by a radial field rf dipole. The computer code ASPIRRIN [4] contains functionality to calculate the spin response function.

Spin-flipping studies of stored polarized beams have been performed at several storage rings, e.g., at the IUCF Cooler using polarized proton beams [5-8] and polarized deuterons [9], and at SHR at MIT-Bates using polarized electrons [10]. Some of the studies employed $\mathrm{rf}$ solenoids and others used radial field rf dipoles. In some cases the spins were flipped in the presence of a nearly full- strength Siberian snake (a solenoid snake in all cases) $[7,8,10]$.

More recently, the SPIN@COSY collaboration has performed numerous spin-flip studies at the COSY Cooler synchrotron, with beams of polarized protons $[11,12]$ and polarized deuterons [13]. A compilation of the data, and theoretical calculations, for spin-flip resonance widths was reported in [11]; large discrepancies between theory and the measurements were noted. More recently, in [14], it was reported that many of the differences for the polarized proton data were due to interference with a nearby intrinsic resonance. The data for the deuterons remains unexplained, and new spin-flip measurements of stored polarized deuteron beams [15] also report experimental measurements of the spin resonance widths which are smaller (by a factor of about 7) than a naive theoretical calculation which does not take into account the contribution of the vertical betatron oscillations. I shall apply the spin response function to the reported data for polarized deuterons, and demonstrate that Kondratenko's formalism can indeed account for the reduction in the observed spinflip resonance widths.

Note that there was some confusion in [11] of a factor of 2 in the definition of the theoretical expression for the resonance strength. This was clarified by Bai, MacKay, and Roser [16], and the statements in [15] indicate that the matter seems fortunately to be resolved.

I first derive the well-known expression for the resonance strength induced by the direct coupling of a radial field $\mathrm{rf}$ dipole to the spin. This will confirm the expression in [16]. I shall perform my calculation by solving for the Fourier harmonics of the perturbing term in the spin-orbit Hamiltonian. I shall then also derive an expression for the coherent vertical orbital oscillation induced by a radial field rf dipole, and its contribution to the spin-orbit coupling. The resulting overall expression for the effective resonance strength will involve not only the spin tune and the rf-dipole parameters, but also the vertical betatron tune.

I treat a simple model of a planar circular ring with smooth focusing. Kondratenko [3] treated a general ma- 
chine lattice, and derived the relevant one-turn spin-orbit integrals. He also evaluated his expressions for the simple case of smooth focussing. The expression I shall obtain below is very similar to the unnumbered displayed equation between Kondratenko's Eqs. (7.13) and (7.14) in [3].

I treat a positively charged particle, of charge $e$ and mass $m$, with velocity $\boldsymbol{\beta} c$. The Lorentz factor is $\gamma=$ $\left(1-\beta^{2}\right)^{-1 / 2}$. The particle momentum is $p=m c \gamma \boldsymbol{\beta}$. The particle spin will be denoted by $\boldsymbol{s}$. The externally prescribed electric and magnetic fields of the accelerator will be denoted by $\boldsymbol{E}$ and $\boldsymbol{B}$, respectively, with subscripts to indicate an oscillating rf field, etc. I employ cgs units, so the Lorentz force is $e(\boldsymbol{E}+\boldsymbol{\beta} \times \boldsymbol{B})$. I treat a planar circular ring of radius $R$. The independent variable is $\theta=s / R$, where $s$ denotes the arclength around the ring circumference. The positive sense of circulation is counterclockwise around the ring. The coordinate basis is $\left\{\boldsymbol{e}_{1}, \boldsymbol{e}_{2}, \boldsymbol{e}_{3}\right\}$, a righthanded orthonormal triad, where $\boldsymbol{e}_{1}$ is radial, $\boldsymbol{e}_{2}$ is longitudinal, and $\boldsymbol{e}_{3}$ is vertical. The radial and vertical particle coordinates are denoted by $x$ and $y$, respectively. The unperturbed spin tune is $\nu_{0} \equiv G \gamma$. Here $G=(g-2) / 2$ is the particle's magnetic moment anomaly. I neglect the beam momentum spread (and/or synchrotron oscillations) below; this is acceptable for the proposed comparison to the COSY Cooler studies. As is standard, I model the radial field rf dipole by a $\delta$-function (this was also done by Kondratenko [3] and by Bai, MacKay, and Roser [16]). This is an excellent approximation in practice.

The spin precession equation, for motion in external transverse $(\perp)$ and longitudinal ( $\|$ ) magnetic fields, is $[17,18]$

$$
\frac{d \boldsymbol{s}}{d t}=-\frac{e}{m c}\left[\left(G+\frac{1}{\gamma}\right) \boldsymbol{B}_{\perp}+\frac{G+1}{\gamma} \boldsymbol{B}_{\|}\right] \times s .
$$

Here the independent variable is the time $t$. In accelerator coordinates, using $\theta$ as the independent variable, the spin precession equation is

$$
\frac{d s}{d \theta}=W \times s,
$$

where, for a planar ring,

$$
\boldsymbol{W}=-R\left[(G \gamma+1) \frac{e \boldsymbol{B}_{\perp}}{p c}+(G+1) \frac{e \boldsymbol{B}_{\|}}{p c}\right]-\boldsymbol{e}_{3} .
$$

For the model of interest below, there is no longitudinal magnetic field, and so

$$
\boldsymbol{W}=-R(G \gamma+1) \frac{e B_{y}}{p c} \boldsymbol{e}_{3}-R(G \gamma+1) \frac{e B_{x}}{p c} \boldsymbol{e}_{1}-\boldsymbol{e}_{3} .
$$

The terms along $\boldsymbol{e}_{3}$ describe spin precession around the vertical axis. The radial field $B_{x}$ comprises the contributions from radial field rf dipole and the ring quadrupoles (vertical orbital oscillations). Now

$$
-R \oint \frac{e B_{y}}{p c} d \theta^{\prime}=2 \pi
$$

and in fact I have already assumed that $B_{y}$ is uniform around the circumference. I shall decompose the term in $B_{x}$ into terms $w_{\text {osc }}$ (for the rf dipole) and $w_{\text {coh }}$ (for the vertical oscillation). Then

$$
\boldsymbol{W}=\nu_{0} \boldsymbol{e}_{3}+w_{\mathrm{osc}} \boldsymbol{e}_{1}+w_{\mathrm{coh}} \boldsymbol{e}_{1}
$$

Representing the spin operator by $\frac{1}{2} \boldsymbol{\sigma}$, i.e., employing a spinor representation, the spin-orbit Hamiltonian is

$$
\mathcal{H}=\frac{1}{2} \boldsymbol{W} \cdot \boldsymbol{\sigma}=\frac{1}{2}\left(\nu_{0} \sigma_{3}+w_{\mathrm{osc}} \sigma_{1}+w_{\mathrm{coh}} \sigma_{1}\right) .
$$

I shall need at some stage to transform to a frame rotating around the vertical. Say the rotation tune is $\bar{\nu}$, then the transformed Hamiltonian is

$$
\begin{aligned}
\overline{\mathcal{H}} & =\frac{1}{2}\left[\left(\nu_{0}-\bar{\nu}\right) \sigma_{3}+e^{i \bar{\nu} \sigma_{3} / 2}\left(w_{\mathrm{osc}}+w_{\mathrm{coh}}\right) \sigma_{1} e^{-i \bar{\nu} \sigma_{3} / 2}\right] \\
& =\frac{1}{2}\left[\left(\nu_{0}-\bar{\nu}\right) \sigma_{3}+e^{i \bar{\nu} \sigma_{3}}\left(w_{\mathrm{osc}}+w_{\mathrm{coh}}\right) \sigma_{1}\right]
\end{aligned}
$$

I shall set $\bar{\nu}=\nu_{0}$ below.

I now calculate the resonance strength for the direct coupling of the radial field $\mathrm{rf}$ dipole to the spin. I follow the notation by Bai, MacKay, and Roser [16]. I model the dipole by a $\delta$-function at the location $\theta_{\text {osc }}$. I write

$$
R B_{x}=B_{\mathrm{osc}} L \cos \left(Q_{\mathrm{osc}} \theta+\chi\right) \delta_{p}\left(\theta-\theta_{\mathrm{osc}}\right) .
$$

Here $B_{\text {osc }}$ is the amplitude of the oscillating radial field, $Q_{\text {osc }}$ is the rf oscillation tune, and $\chi$ is an initial phase. The periodic $\delta$-function is

$$
\delta_{p}\left(\theta-\theta_{\mathrm{osc}}\right)=\sum_{m=-\infty}^{\infty} \delta\left(\theta-\theta_{\mathrm{osc}}-2 \pi m\right) .
$$

Since $\delta_{p}$ is real, its Fourier decomposition can be written in the equivalent forms

$$
\begin{aligned}
\delta_{p}\left(\theta-\theta_{\mathrm{osc}}\right) & =\frac{1}{2 \pi} \sum_{k=-\infty}^{\infty} e^{i k\left(\theta-\theta_{\mathrm{osc}}\right)} \\
& =\frac{1}{2 \pi} \sum_{k=-\infty}^{\infty} e^{-i k\left(\theta-\theta_{\mathrm{osc}}\right)}
\end{aligned}
$$

Both versions will be used below. Then

$$
w_{\mathrm{osc}}=-(G \gamma+1) \frac{e B_{\mathrm{osc}} L}{p c} \cos \left(Q_{\mathrm{osc}} \theta+\chi\right) \delta_{p}\left(\theta-\theta_{\mathrm{osc}}\right) .
$$

Its Fourier decomposition is 


$$
\begin{aligned}
w_{\mathrm{osc}}= & -\frac{1}{2}(G \gamma+1) \frac{e B_{\mathrm{osc}} L}{p c}\left[e^{i\left(Q_{\mathrm{osc}} \theta+\chi\right)}\right. \\
& \left.+e^{-i\left(Q_{\mathrm{osc}} \theta+\chi\right)}\right] \delta_{p}\left(\theta-\theta_{\mathrm{osc}}\right) \\
= & -\frac{G \gamma+1}{4 \pi} \frac{e B_{\mathrm{osc}} L}{p c} \sum_{k=-\infty}^{\infty}\left[e^{i\left[\left(Q_{\mathrm{osc}}-k\right) \theta+k \theta_{\mathrm{osc}}+\chi\right]}\right. \\
& +e^{\left.-i\left[\left(Q_{\mathrm{osc}}-k\right) \theta+k \theta_{\mathrm{osc}}+\chi\right]\right]} \\
= & -\frac{G \gamma+1}{2 \pi} \frac{e B_{\mathrm{osc}} L}{p c} \sum_{k=-\infty}^{\infty} \cos \psi_{k} .
\end{aligned}
$$

In the last line I introduced a symbol which will appear frequently below:

$$
\psi_{k} \equiv\left(Q_{\mathrm{osc}}-k\right) \theta+k \theta_{\mathrm{osc}}+\chi .
$$

The next step is to transform to the rotating frame (setting $\left.\bar{\nu}=\nu_{0}\right)$ as follows:

$$
\begin{aligned}
\overline{\boldsymbol{w}}_{\mathrm{osc}} \cdot \boldsymbol{\sigma}= & e^{i \nu_{0} \theta \sigma_{3}} w_{\mathrm{osc}} \sigma_{1} \\
= & -\frac{G \gamma+1}{4 \pi} \frac{e B_{\mathrm{osc}} L}{p c} e^{i \nu_{0} \theta \sigma_{3}} \\
& \times \sum_{k=-\infty}^{\infty}\left[e^{-i \psi_{k} \sigma_{3}}+e^{i \psi_{k} \sigma_{3}}\right] \sigma_{1} .
\end{aligned}
$$

This is the standard decomposition of a radial field into counterrotating circular fields. Define $\sigma_{ \pm}=\sigma_{1} \pm i \sigma_{2}$. The terms

$$
\begin{aligned}
e^{-i\left(\psi_{k}-\nu_{0} \theta\right) \sigma_{3}} \sigma_{1} & =\Re\left\{e^{-i\left(\psi_{k}-\nu_{0} \theta\right)} \sigma_{+}\right\} \\
& =\cos \left(\psi_{k}-\nu_{0} \theta\right) \sigma_{1}+\sin \left(\psi_{k}-\nu_{0} \theta\right) \sigma_{2} \\
e^{i\left(\psi_{k}+\nu_{0} \theta\right) \sigma_{3}} \sigma_{1} & =\Re\left\{e^{i\left(\psi_{k}+\nu_{0} \theta\right)} \sigma_{-}\right\} \\
& =\cos \left(\psi_{k}+\nu_{0} \theta\right) \sigma_{1}-\sin \left(\psi_{k}+\nu_{0} \theta\right) \sigma_{2}
\end{aligned}
$$

describe, respectively, fields rotating counterclockwise and clockwise around the vertical axis. Then

$$
\begin{aligned}
\overline{\boldsymbol{w}}_{\mathrm{osc}} \cdot \boldsymbol{\sigma}= & -\frac{G \gamma+1}{4 \pi} \frac{e B_{\mathrm{osc}} L}{p c} \\
& \times \sum_{k=-\infty}^{\infty} \Re\left\{e^{-i\left[\left(Q_{\mathrm{osc}}-\nu_{0}-k\right) \theta+k \theta_{\mathrm{osc}}+\chi\right]} \sigma_{+}\right. \\
& \left.+e^{i\left[\left(Q_{\mathrm{osc}}+\nu_{0}-k\right) \theta+k \theta_{\mathrm{osc}}+\chi\right] \sigma_{3}} \sigma_{-}\right\} \\
\equiv & \sum_{k=-\infty}^{\infty} \mathfrak{R}\left\{\epsilon_{k}^{+} e^{-i\left(Q_{\mathrm{osc}}-\nu_{0}-k\right) \theta} \sigma_{+}\right. \\
& \left.+\epsilon_{k}^{-} e^{i\left(Q_{\mathrm{osc}}+\nu_{0}-k\right) \theta} \sigma_{-}\right\} .
\end{aligned}
$$

The next step is to make the approximation that the resonances induced by the individual harmonics are well separated. One then selects only the harmonic which is closest to zero. This harmonic is slowly varying while the others oscillate rapidly and average to zero (but see below).
Suppose that the slowest varying harmonic is one of the $\epsilon_{k}^{+}$terms, and that $Q_{\text {osc }}-\nu_{0}-k$ is closest to zero when $k=j$. Then

$$
\overline{\boldsymbol{w}}_{\mathrm{osc}} \cdot \boldsymbol{\sigma} \simeq \Re\left\{\boldsymbol{\epsilon}_{j}^{+} e^{i\left(Q_{\mathrm{osc}}-\nu_{0}-j\right) \theta} \sigma_{+}\right\} .
$$

A similar expression occurs if the slowly varying term is $\epsilon_{j}^{-}$instead. The resonances driven by the $\epsilon_{j}^{ \pm}$terms are $Q_{\text {osc }}=j \pm \nu_{0}$, respectively. The resonance strength is the amplitude of the relevant harmonic:

$$
\left|\epsilon_{j}^{ \pm}\right|=\left|\frac{G \gamma+1}{4 \pi} \frac{e B_{\mathrm{osc}} L}{p c}\right| .
$$

This agrees with the expression derived in [16].

I comment briefly on the validity of the averaging over the "rapidly varying" Fourier harmonics. The terms in $\epsilon_{k}^{-}$ drive the "mirror resonances" $Q_{\mathrm{osc}}=k-\nu_{0}$. In general, the spectra of resonances and mirrors do not coincide. This is not so, however, if the value of the fractional spin tune $[\nu]$ is either 0 or $\frac{1}{2}$. Then there will be two slowly varying terms in the spin-orbit Hamiltonian, say $\epsilon_{j}^{+}$and $\epsilon_{j^{\prime}}^{-}$. The magnitudes of the Fourier harmonics are equal: $\left|\epsilon_{j}^{+}\right|=$ $\left|\epsilon_{j^{\prime}}^{-}\right|$. Both resonances are driven simultaneously, but this does not mean that the resonance strength is doubled. Instead, it means that the averaging which was implicit in the above derivation for the resonance strength is invalid. As the rf-dipole frequency is swept, the resonance and its mirror are crossed in opposite directions, so what really happens is that the adiabatic condition for spin flipping cannot be achieved. This circumstance was noted already by Koop and Shatunov [19], who worked out the theory for spin flipping in a ring with Siberian snake(s).

I now treat the second contribution to the depolarization: an oscillating radial dipole field can also drive a coherent vertical orbital oscillation. One must solve Hill's equation with a driving term:

$$
\frac{d^{2} y}{d \theta^{2}}+G_{y} y=-R^{2} \frac{e B_{x}}{p c} .
$$

Here $G_{y}$ is the focusing function

$$
G_{y}=-R^{2} \frac{e}{p c} \frac{\partial B_{y}}{\partial x} .
$$

I treat a smooth focusing model, so

$$
G_{y}=Q^{2} \text {. }
$$

Naively, one expects that $Q$ is the vertical betatron tune, but one should not be hasty on this point. Since the rf dipole is being modeled as a point object, one should really solve for the coherent oscillation $y_{\text {coh }}$ using a one-turn map from $\theta_{\text {osc }}$ to $\theta_{\text {osc }}+2 \pi$ followed by a kick to $d y / d \theta$, followed by another one-turn map plus kick, etc. In this scenario, the value of $Q$ is aliased to the betatron tune plus an integer. I proceed with the differential equation formalism. Then 


$$
\begin{aligned}
\frac{d^{2} y}{d \theta^{2}}+Q^{2} y & =-R \frac{e B_{\mathrm{osc}} L(\theta)}{p c} \delta_{p}\left(\theta-\theta_{\mathrm{osc}}\right) \\
& =-\frac{R}{4 \pi} \frac{e B_{\mathrm{osc}} L}{p c} \sum_{k=-\infty}^{\infty}\left(e^{-i \psi_{k}}+e^{i \psi_{k}}\right) .
\end{aligned}
$$

This can be solved by linear superposition, by writing $y_{\text {coh }}=y^{+}+y^{-}$, where

$$
y^{ \pm}=\sum_{k=-\infty}^{\infty} y_{k}^{ \pm}
$$

and

$$
\frac{d^{2} y_{k}^{ \pm}}{d \theta^{2}}+Q^{2} y_{k}^{ \pm}=-\frac{R}{4 \pi} \frac{e B_{\mathrm{osc}} L}{p c} e^{\mp i \psi_{k}} .
$$

The solution is

$$
y_{\mathrm{coh}}=-\frac{R}{4 \pi} \frac{e B_{\mathrm{osc}} L}{p c} \sum_{k=-\infty}^{\infty} \frac{e^{-i \psi_{k}}+e^{i \psi_{k}}}{Q^{2}-\left(Q_{\mathrm{osc}}-k\right)^{2}} .
$$

The contribution of the coherent vertical orbital oscillation to the spin precession occurs via the radial fields in the ring quadrupoles:

$$
w_{\mathrm{coh}}=-R(G \gamma+1) \frac{e}{p c} B_{\mathrm{quad}} .
$$

In a smooth focusing model

$$
w_{\mathrm{coh}}=-(G \gamma+1) \frac{Q^{2}}{R} y_{\mathrm{coh}} .
$$

Using the expression for the orbital oscillation derived above,

$$
w_{\mathrm{coh}}=Q^{2} \frac{G \gamma+1}{4 \pi} \frac{e B_{\mathrm{osc}} L}{p c} \sum_{k=-\infty}^{\infty} \frac{e^{-i \psi_{k}}+e^{i \psi_{k}}}{Q^{2}-\left(Q_{\mathrm{osc}}-k\right)^{2}} .
$$

The total spin response is

$$
\begin{aligned}
w_{\text {resp }}= & w_{\mathrm{osc}}+w_{\mathrm{coh}} \\
= & -\frac{G \gamma+1}{4 \pi} \frac{e B_{\mathrm{osc}} L}{p c} \sum_{k=-\infty}^{\infty}\left(e^{-i \psi_{k}}+e^{i \psi_{k}}\right) \\
& +Q^{2} \frac{G \gamma+1}{4 \pi} \frac{e B_{\mathrm{osc}} L}{p c} \sum_{k=-\infty}^{\infty} \frac{e^{-i \psi_{k}}+e^{i \psi_{k}}}{Q^{2}-\left(Q_{\mathrm{osc}}-k\right)^{2}} .
\end{aligned}
$$

This can be simplified to

$$
\begin{aligned}
w_{\text {resp }}= & -\frac{G \gamma+1}{4 \pi} \frac{e B_{\mathrm{osc}} L}{p c} \sum_{k=-\infty}^{\infty}\left(e^{-i \psi_{k}}+e^{i \psi_{k}}\right) \\
& \times \frac{\left(Q_{\mathrm{osc}}-k\right)^{2}}{\left(Q_{\mathrm{osc}}-k\right)^{2}-Q^{2}} .
\end{aligned}
$$

One can now transform to the rotating frame, etc. It is clear that the resonance strengths are similar to before, but multiplied by a factor which depends on $k$ and the vertical betatron tune. The resonance coefficients are

$$
\bar{w}_{k}^{ \pm}=\epsilon_{k}^{ \pm} \frac{\left(Q_{\mathrm{osc}}-k\right)^{2}}{\left(Q_{\mathrm{osc}}-k\right)^{2}-Q^{2}} .
$$

The technical definition of the resonance strength is obtained by setting the rf-dipole frequency to the center of the resonance. As before, suppose the slowly varying harmonic corresponds to $k=j$, then set $Q_{\text {osc }}-j= \pm \nu_{0}$ (for an $\epsilon_{j}^{ \pm}$term, respectively). The effective resonance strength is

$$
\left|\bar{w}_{j}^{ \pm}\right|=\left|\epsilon_{j}^{ \pm}\right|\left|\frac{\nu_{0}^{2}}{\nu_{0}^{2}-Q^{2}}\right|
$$

I now compare the theoretical calculation to the measurements reported in [13]. A summary of the comparison is displayed in Table I. The deuteron beam was accelerated to a desired final energy and the beam then coasted at flattop (the rf cavities were shorted). Hence, there were no synchrotron oscillations, which justifies the neglect of synchrotron oscillations in the above theoretical calculations. From [13], the vertical betatron tune was $Q_{y}=3.6$ and the relative momentum spread was $\left(\sigma_{p} / p\right)_{\mathrm{rms}}=5 \times$ $10^{-4}$. I shall treat the deuteron beam as monoenergetic. The flattop momentum was reported as $1.85 \mathrm{GeV} / c$, and the spin tune was $\nu_{0}=G \gamma=-0.20084$, which is negative because for deuterons $G_{d} \simeq-0.142987$. The rf-dipole field integral was $(0.54 \pm 0.03) \mathrm{T}-\mathrm{mm}(\mathrm{rms})$, so one can calculate

$$
\begin{aligned}
\epsilon_{\mathrm{th}} & =\frac{-0.20084+1}{4 \pi} \frac{\sqrt{2} \times(0.54 \pm 0.03) \times 10^{-3}}{1.85 \times 3.3356} \\
& =(7.9 \pm 0.4) \times 10^{-6}
\end{aligned}
$$

The information in [13] gave the theoretical value (divided by 2 ; see my statement above about a temporary confusion concerning factors of 2 ) of

$$
\epsilon_{\mathrm{th}}=(8 \pm 0.5) \times 10^{-6}
$$

TABLE I. Comparison of experimental and theoretical resonance strengths (including the spin response function) for the spin flipping of stored polarized deuteron beams.

\begin{tabular}{lccccrr}
\hline \hline Particle & Resonance & $G \gamma$ & $Q$ & $\epsilon_{\mathrm{th}}\left(\times 10^{6}\right)$ & $\bar{w}_{\mathrm{th}}\left(\times 10^{6}\right)$ & $\epsilon_{\mathrm{exp}}\left(\times 10^{6}\right)$ \\
\hline Deuteron & $1+G \gamma$ & -0.20084 & 0.6 & $8 \pm 0.5$ & $1.0 \pm 0.06$ & $1.165 \pm 0.014(\mathrm{vector})$ \\
& & & & & $1.155 \pm 0.027$ (tensor) \\
\hline \hline
\end{tabular}


which is very close to Eq. (34). I use the value in Eq. (35) below. The experimental studies for the spin flip of the vector and tensor deuteron polarizations yielded the same resonance strength, within the experimental precision. (One expects this to be the case from angular momentum theory, but experiments must check such details.) The experimentally measured values of the resonance strengths were [13]

$$
\begin{aligned}
& \epsilon_{V}=(1.165 \pm 0.014) \times 10^{-6} \\
& \epsilon_{T}=(1.155 \pm 0.027) \times 10^{-6} .
\end{aligned}
$$

The rf-dipole frequency was aliased to the resonance $1+$ $G \gamma$, so $Q_{\text {osc }}=1+\nu_{0} \simeq 0.8$ so I alias $Q=Q_{y}-3=0.6$. Then

$$
\frac{\nu_{0}^{2}}{\nu_{0}^{2}-Q^{2}} \simeq \frac{(-0.20084)^{2}}{(-0.20084)^{2}-(0.6)^{2}} \simeq-0.126
$$

and the effective resonance strength is

$$
\bar{w}_{\mathrm{th}} \simeq(1.0 \pm 0.06) \times 10^{-6}
$$

which is remarkably close to the experimental value.

I make some further observations:

(i) More recent deuteron spin-flip studies reported in [15], also at a vertical betatron tune of 3.6, also yielded experimental widths about a factor of 7 smaller than the naive theoretical calculation (i.e. without the inclusion of the spin response function). This is consistent with the above finding of -0.126 .

(ii) It is also stated in [15] that deuteron spin-flip measurements made previously at the IUCF Cooler [9] gave a data/theory ratio close to 1 . However, it was also stated that the IUCF Cooler spin-flip studies of polarized deuterons employed an $r f$ solenoid. A solenoid (longitudinal magnetic field) does not excite vertical betatron oscillations, so the multiplier from the spin response function is unity for an $\mathrm{rf}$ solenoid. Hence, it is a pleasant confirmation of Kondratenko's spin response formalism [3] that the IUCF Cooler studies reported a data/theory ratio close to unity.

(iii) Additional studies reported in [15] measured the data/theory ratio of resonance width as a function of the vertical betatron tune (see Fig. 4 of [15]). The experimenters fitted the data/theory ratio using a functional form

$$
r=A+\frac{B}{\nu_{r}-\nu_{y}},
$$

where $\nu_{r} \simeq 3.798$ [15] is the resonant tune and $\nu_{y}$ is the vertical betatron tune. This is not too different from the simple functional form I derived in Eq. (33). The presence of a nondiverging term $A$ is quite natural, if one employs a more detailed model than I have done.

To conclude, the polarized deuteron spin-flip data at both the COSY Cooler [13,15] and the IUCF Cooler [9] support the validity of Kondratenko's spin response formalism [3] for resonance widths. In the case of the IUCF Cooler measurements, the use of an rf solenoid indicates that the naive theory is indeed satisfactory when vertical betatron oscillations are not excited by the spin flipper. The model I have developed above is very simple. A more detailed treatment of the COSY Cooler machine lattice should be able to explain the other data reported in [15] (e.g. sweep of vertical betatron tune) which I have not treated above.

\section{ACKNOWLEDGMENTS}

I thank Yuri M. Shatunov for bringing the spin response formalism to my attention, also the ASPIRRIN computer code, and for general education about spin flippers.

[1] M. Froissart and R. Stora, Nucl. Instrum. Methods Phys. Res., Sect. A 7, 297 (1960) (in French).

[2] L. Arnaudon et al., Phys. Lett. B 284, 431 (1992).

[3] A. M. Kondratenko, Novosibirsk Report No. 82-28, 1982.

[4] E. A. Perevedentsev, Yu. M. Shatunov, and V. Ptitsin, Proceedings of SPIN02, AIP Conf. Proc. No. 675 (American Institute of Physics, New York, 2003), p. 761.

[5] D. D. Caussyn et al., Phys. Rev. Lett. 73, 2857 (1994).

[6] V.A. Anferov et al., Phys. Rev. ST Accel. Beams 3, 041001 (2000).

[7] B. B. Blinov et al., Phys. Rev. Lett. 81, 2906 (1998).

[8] B. B. Blinov et al., Phys. Rev. ST Accel. Beams 3, 104001 (2000).

[9] V.S. Morozov et al., Phys. Rev. Lett. 91, 214801 (2003).

[10] V.S. Morozov et al., Phys. Rev. ST Accel. Beams 4, 104002 (2001).

[11] V.S. Morozov et al., Phys. Rev. ST Accel. Beams 7, 024002 (2004).

[12] M. A. Leonova et al., Phys. Rev. Lett. 93, 224801 (2004).

[13] V.S. Morozov et al., Phys. Rev. ST Accel. Beams 8, 061001 (2005).

[14] M. A. Leonova et al., Phys. Rev. ST Accel. Beams 9, 051001 (2006).

[15] A.D. Krisch et al., Phys. Rev. ST Accel. Beams 10, 071001 (2007).

[16] M. Bai, W. W. MacKay, and T. Roser, Phys. Rev. ST Accel. Beams 8, 099001 (2005).

[17] L. H. Thomas, Philos. Mag. 3, 1 (1927).

[18] V. Bargmann, L. Michel, and V.L. Telegdi, Phys. Rev. Lett. 2, 435 (1959).

[19] I. Koop and Yu. M. Shatunov, Proceedings of SPIN94, AIP Conf. Proc. No. 343 (American Institute of Physics, New York, 1995), p. 317. 